\title{
EVALUACIÓN ERGONÓMICA DEL PUESTO DE TRABAJO DE UN ACADÉMICO DE EDUCACIÓN SUPERIOR Y SUS ACTIVIDADES EXTRAESCOLARES
}

\author{
ERGONOMIC EVALUATION OF THE JOB OF A \\ ACADEMIC OF HIGHER EDUCATION AND ITS EXTRACURRICULAR ACTIVITIES
}

\author{
Gilberto Chávez-Esquivel* \\ Brenda Crystal Suárez-Espinosa** \\ Francisco Jesús Arévalo-Carrasco ${ }^{* * *}$ \\ Aarón Guerrero-Campanur ${ }^{* * * *}$
}

\begin{abstract}
Resumen: Se realizó una evaluación ergonómica del puesto de trabajo de un académico de educación superior. Mediante un estudio de tiempos se determinó la carga organizacional y se estimó el porcentaje de carga cardiovascular para conocer las exigencias laborales. El trabajo administrativo fue evaluado mediante el método ROSA, identificándose mejoras para una postura de trabajo óptima. Se obtuvieron los ángulos de las posturas adoptadas durante el trabajo administrativo y se comparó la desviación que existe entre las condiciones de trabajo y lo que se considera como aceptable. Se midieron los niveles de iluminación en el espacio evaluado para determinar si la distribución de la luz es adecuada aplicando el criterio de uniformidad de iluminación. El estudio de tiempos mostró porcentajes mayores al $80 \%$ en la suma de las actividades principales y secundarias, considerado como sobredemanda laboral. Como promedio de la jornada laboral los porcentajes de carga cardiovascular no superan el 40\%, lo que si bien, no es trabajo pesado, para el caso de estudio las jornadas laborales superan las 8 horas diarias. La desviación de las características del puesto evaluado demuestra sobrecarga postural, y el nivel de actuación obtenido mediante el método ROSA, sugiere intervención inmediata. Las luminarias utilizadas no proveen el mínimo requerido de iluminancia y la uniformidad de esta no es adecuada. Las recomendaciones principales están orientadas a mejorar los ángulos del equipo de cómputo, así como los periféricos, aunado a utilizar tanto sillas como escritorios con las dimensiones favorables, implementación de luz artificial y mayor aprovechamiento de la luz natural.
\end{abstract}

Palabras clave: Estudio de tiempos, carga cardiovascular, fatiga postural, iluminación.

Abstract: An ergonomic evaluation of the job position of a higher education academic was carried out. By means of a time study, the organizational load was determined and the percentage of cardiovascular load was estimated to know the work demands. Administrative work was evaluated

\footnotetext{
*Instituto Tecnológico Superior de Uruapan, Tecnológico Nacional de México. Uruapan, México. Correo electrónico: gilberto.ce@uruapan.tecnm.mx. Orcid: https://orcid.org/oooo-ooo2-2896-1239. Autor de correspondencia.

** Instituto Tecnológico Superior de Uruapan, Tecnológico Nacional de México. Uruapan, México. Correo electrónico: brenda.se@uruapan.tecnm.mx. Orcid: https://orcid.org/oooo-0oo2-1371-3267

***Instituto Tecnológico Superior de Uruapan, Tecnológico Nacional de México. Uruapan, México. Correo electrónico: francisco.ac@uruapan.tecnm.mx. Orcid: https://orcid.org/oooo-0002-7578-0705

****Instituto Tecnológico Superior de Uruapan, Tecnológico Nacional de México. Uruapan, México. Correo electrónico: aaron.gc@uruapan.tecnm.mx. Orcid: https://orcid.org/oooo-0002-9034-045X
} 
using the ROSA method, identifying improvements for an optimal work posture. The angles of the postures adopted during the administrative work were obtained and the deviation that exists between the working conditions and what is considered acceptable was compared. The illumination levels in the evaluated space were measured to determine if the light distribution is adequate by applying the illumination uniformity criterion. The time study showed percentages greater than $80 \%$ in the sum of the main and secondary activities, considered as labor over-demand. As an average of the working day, the percentages of cardiovascular load do not exceed $40 \%$, which although it is not heavy work, for the case study the working hours exceed 8 hours a day. The deviation of the characteristics of the evaluated position shows postural overload, and the level of performance obtained through the ROSA method suggests immediate intervention. The luminaires used do not provide the minimum required illuminance and its uniformity is not adequate. The main recommendations are aimed at improving the angles of the computer equipment, as well as the peripherals, coupled with the use of both chairs and desks with favorable dimensions, implementation of artificial light and greater use of natural light.

Keywords: Time study, cardiovascular load, postural fatigue, lighting.

Recepción: 08.07.2021 / Revisión: 07.09.2021 / Aceptación: 28.10.2021

\section{Introducción}

Con la presencia del coronavirus SARS-Cov-2 que surgió en China, y que posteriormente se extendió a todos los continentes del mundo provocando una pandemia, siendo América uno de los continentes más afectados (Gobierno de México, 2020, 28 de Octubre), existió la necesidad de modificar los hábitos de trabajo. Uno de los nuevos hábitos instaurados sin duda fue el teletrabajo, sobre todo en aquellos de tipo administrativo, modalidad que no se implementó, en algunos casos, con el cumplimiento de requisitos de diseño adecuados, considerando el equilibrio entre las demandas laborales y las capacidades de las personas. La disciplina científica relacionada con la comprensión de estas interacciones es la ergonomía (Departamento de Ergonomía de la Universidad de Concepción y Sociedad Chilena de Ergonomía, 2020).

Uno de los aspectos más críticos a la hora de valorar la carga física de un puesto de trabajo corresponde a la evaluación de las posturas de trabajo, en parte debido a que este factor influye en otros, como los esfuerzos musculares, la actividad física y la seguridad del puesto de trabajo (Maestre, 2015). Al respecto, algunos de los factores de riesgo más comunes del trabajo administrativo derivan de la permanencia en posición sedente durante largo tiempo, que si se mantiene incorrectamente, incrementa la fatiga muscular, y por otra parte, del empleo del teclado y el mouse; por ejemplo, movimientos repetitivos de los dedos, las manos y las muñecas, mantenimiento del antebrazo y la muñeca en posturas incómodas o presión de contacto elevada en la muñeca que maneja el mouse (Diego-Mas, 2015).

Para la eficacia de la prevención es necesario entender lo que significa la postura, la cual es el resultado de una decisión, que busca una eficacia máxima y una seguridad óptima para la salud del trabajador (Álvarez, 2009). Existe evidencia epidemiológica que indica que determinadas posturas de trabajo pueden generar trastornos músculo-esqueléticos (TME's). De este modo, el término sobrecarga postural está referido a posiciones adoptadas por los 
segmentos corporales, que puedan implicar riesgo para la integridad y función del sistema músculo-esquelético (Pheasant, 1998). Estudios indican que la prevalencia de los TME's en puestos de oficina oscila entre el $10 \%$ y el $62 \%$, generalmente relacionados con las extremidades superiores, cuello y espalda (Diego-Mas, 2015). Los TME's también se presentan por una elevada carga de trabajo, por la correlación con la fatiga física.

La frecuencia cardíaca es un excelente indicador fisiológico de esfuerzo sobre el sistema cardiovascular. Los latidos cardíacos no solo aumentan por el trabajo físico desplegado, sino también como una forma de ayudar a eliminar el calor corporal, para evitar que suba la temperatura del organismo. Por tal motivo, se producen ajustes para que la sangre fluya (refrigeración) más rápido y uno de estos es el aumento de la frecuencia cardíaca (Apud \& Meyer, 2009).

Aunado a los problemas de posturas, en las actividades del trabajo administrativo en ocasiones no se tienen los niveles mínimos de iluminación que deben incidir en el plano de trabajo, para cada tipo de tarea visual o área de trabajo. El reconocimiento de las condiciones de iluminación, tiene el propósito de identificar las tareas visuales asociadas a los puestos de trabajo, examinando aquellas en donde exista una iluminación deficiente o en exceso que provoque deslumbramientos (NOM-025-STPS-2008). Todo lugar de trabajo, con excepción de faenas mineras subterráneas o similares, deberá estar iluminando con luz natural o artificial dependiendo de la faena a realizar, dicha iluminación es expresada en (Lx) luxes (Decreto Supremo 594, 1999).

\section{Objetivo general}

Evaluar ergonómicamente el puesto de trabajo de un profesor de educación superior, así como las actividades que realiza fuera de las responsabilidades docentes, para optimizar las condiciones de trabajo.

\section{Objetivos específicos}

- Evaluar la carga organizacional de trabajo y el esfuerzo cardiovascular.

- Estudiar las características del mobiliario y posturas adoptadas durante el trabajo de tipo administrativo.

- Obtener los niveles de iluminación del puesto de trabajo.

- Otorgar recomendaciones orientadas a mejorar las condiciones disergonómicas.

\section{Materiales y métodos}

Para comprender las actividades del puesto de trabajo se realizó un estudio de tiempos. El estudio de tiempos ayudó a conocer la carga organizacional de trabajo, desde las labores del trabajador, el origen de las demandas del sistema y la interacción puesto de trabajo-persona. Por otra parte, se evaluaron las asignaciones de funciones, las exigencias que impone la organización para que se ejecuten las tareas durante la jornada laboral (Organización Internacional del Trabajo, 1998). 
Dado que, frecuentemente las exigencias laborales conllevan a alteraciones fisiológicas en los trabajadores, para determinar si las demandas impuestas están dentro de los límites razonables de carga física, se llevó a cabo la medición de los latidos cardíacos, expresados como porcentaje de carga cardiovascular, calculada con la fórmula:

$\% \mathrm{CC}=(\mathrm{FC}$ trabajo- $\mathrm{FC}$ reposo $) /(\mathrm{FC}$ máxima- $\mathrm{FC}$ reposo $) \times 100$

Donde:

\% CC: Porcentaje de carga cardiovascular.

FC trabajo: Frecuencia cardíaca de trabajo.

FC reposo: Frecuencia cardíaca de reposo.

FC máxima: Frecuencia cardíaca máxima teórica, obtenida de la diferencia entre 220 y la edad.

Se considera que el trabajo es pesado cuando en promedio de una jornada de 8 horas se excede el $40 \%$ de la CC (Apud \& Meyer, 2009).

Para la obtención de los latidos cardíacos, se utilizó un pulsómetro M20o de marca Polar, el cual cuenta con monitor de frecuencia cardíaca, sin embargo, para mejorar la precisión de la frecuencia cardíaca se adicionó un sensor por medio la banda telemétrica Polar H1O. Con ayuda del pulsómetro M20o se dio seguimiento a la quema de calorías durante las labores del trabajador, obteniéndose el gasto energético por día.

El trabajador utilizó el pulsómetro M20o en su muñeca izquierda y la banda telemétrica en la parte torácica del cuerpo. Previo a la utilización del pulsómetro M20o, este se programó en la plataforma de Polar Flow capturando los datos personales del trabajador.

El trabajo administrativo se evaluó mediante el método ROSA (Rapid Office Strain Assessment). Este método emplea diagramas de puntuación asignando un valor a cada uno de los elementos del puesto: silla, pantalla, teclado, mouse y teléfono. Obtenidos los datos necesarios se puntuaron los diferentes elementos del puesto con ayuda de los diagramas de puntuación, los cuales fueron diseñados para asignarle la puntuación de 1 si la situación de un elemento del puesto es la ideal; conforme la situación del elemento se desvía de la ideal la puntuación crece de forma lineal hasta 3. Por otra parte, ciertas situaciones específicas respecto a cada elemento incrementan la puntuación obtenida por el elemento $(+1)$. Una vez obtenidas las puntuaciones de los cinco elementos del puesto considerados, se obtuvieron las puntuaciones parciales y la puntuación final. La puntuación final puede oscilar entre 1 y 10, siendo más grande cuanto mayor es el riesgo para la persona que ocupa el puesto (DiegoMas, 2015).

La figura 1 muestra los niveles de actuación según la puntuación final del método ROSA. 
Figura 1. Niveles de actuación del método ROSA.

\begin{tabular}{|c|c|l|l|}
\hline Puntuación & Riesgo & Nivel & Actuación \\
\hline 1 & Inapreciable & 0 & No es necesaria actuación. \\
\hline $2-3-4$ & Mejorable & 1 & Pueden mejorarse algunos elementos del puesto. \\
\hline 5 & Alto & 2 & Es necesaria la actuación. \\
\hline $6-7-8$ & Muy Alto & 3 & Es necesaria la actuación cuanto antes. \\
\hline $9-10$ & Extremo & 4 & Es necesaria la actuación urgentemente. \\
\hline
\end{tabular}

Fuente. Diego-Mas (2015).

Esta evaluación se complementó con fotografías, y tras la observación, con una breve entrevista al trabajador para aclarar aspectos de la tarea y el puesto de trabajo.

La suma de las puntuaciones de la altura del asiento y la profundidad del asiento, además, la suma de las puntuaciones de los reposabrazos y el respaldo, se emplean para obtener el valor correspondiente de la figura 2. A la puntuación obtenida se le sumó la puntuación correspondiente al tiempo de uso de la silla.

A la puntuación obtenida para la pantalla se le agregó la puntuación debida al tiempo de uso del monitor. La suma de ambas puntuaciones determinó la puntuación del monitor. De la misma manera, a la puntuación obtenida para el teléfono se le adicionó a la puntuación debida al tiempo de uso, pero considerando ahora el tiempo que el trabajador emplea el teléfono. La suma de ambas puntuaciones determinó la puntuación del teléfono. Ambas puntuaciones, la del teléfono y la del monitor, se emplearon para obtener el valor correspondiente de la figura 3 .

En la puntuación obtenida para el mouse se adicionó la puntuación correspondiente al tiempo de uso del mouse. La suma de ambas puntuaciones determinó la puntuación del mouse. De la misma manera, a la puntuación obtenida para el teclado es necesario aumentar la puntuación debida al tiempo obtenido del uso del teclado, pero considerando ahora el tiempo que el trabajador emplea el teclado. La suma de ambas puntuaciones determinó la puntuación del teclado. Ambas puntuaciones, la del mouse y la del teclado, se emplean para obtener el valor correspondiente de la figura 4.

Finalmente, se obtuvo la puntuación de la pantalla y los periféricos. Para ello, se utilizó la figura 5. Para consultar esta tabla se emplean los valores obtenidos anteriormente de la figura 3 y de la figura 4.

Una vez obtenidas la puntuación de la silla, la puntuación de la pantalla y los periféricos se emplea la figura 6. 
Figura 2. Diagrama de puntuación tabla A del método ROSA.

\begin{tabular}{|c|c|c|c|c|c|c|c|c|c|}
\hline \multirow{2}{*}{\multicolumn{2}{|c|}{ TABLAA }} & \multicolumn{8}{|c|}{ Altura del Asiento + Profundidad del Asiento } \\
\hline & & 2 & 3 & 4 & 5 & 6 & 7 & 8 & 9 \\
\hline \multirow{7}{*}{ 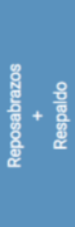 } & 2 & 2 & 2 & 3 & 4 & 5 & 6 & 7 & 8 \\
\hline & 3 & 2 & 2 & 3 & 4 & 5 & 6 & 7 & 8 \\
\hline & 4 & 3 & 3 & 3 & 4 & 5 & 6 & 7 & 8 \\
\hline & 5 & 4 & 4 & 4 & 4 & 5 & 6 & 7 & 8 \\
\hline & 6 & 5 & 5 & 5 & 5 & 6 & 7 & 8 & 9 \\
\hline & 7 & 6 & 6 & 6 & 7 & 7 & 8 & 8 & 9 \\
\hline & 8 & 7 & 7 & 7 & 8 & 8 & 9 & 9 & 9 \\
\hline
\end{tabular}

Fuente. Diego-Mas (2015).

Figura 3. Diagrama de puntuación tabla B del método ROSA.

\begin{tabular}{|c|c|c|c|c|c|c|c|c|c|}
\hline \multirow{2}{*}{\multicolumn{2}{|c|}{ TABLA B }} & \multicolumn{8}{|c|}{ Puntuación de la Pantalla } \\
\hline & & 0 & 1 & 2 & 3 & 4 & 5 & 6 & 7 \\
\hline \multirow{7}{*}{ 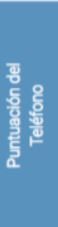 } & 0 & 1 & 1 & 1 & 2 & 3 & 4 & 5 & 6 \\
\hline & 1 & 1 & 1 & 2 & 2 & 3 & 4 & 5 & 6 \\
\hline & 2 & 1 & 2 & 2 & 3 & 3 & 4 & 6 & 7 \\
\hline & 3 & 2 & 2 & 3 & 3 & 4 & 5 & 6 & 8 \\
\hline & 4 & 3 & 3 & 4 & 4 & 5 & 6 & 7 & 8 \\
\hline & 5 & 4 & 4 & 5 & 5 & 6 & 7 & 8 & 9 \\
\hline & 6 & 5 & 5 & 6 & 7 & 8 & 8 & 9 & 9 \\
\hline
\end{tabular}

Fuente. Diego-Mas (2015).

Figura 4. Diagrama de puntuación tabla C del método ROSA.

\begin{tabular}{|c|c|c|c|c|c|c|c|c|c|}
\hline \multirow{2}{*}{\multicolumn{2}{|c|}{ TABLAC }} & \multicolumn{8}{|c|}{ Puntuacion del Teclado } \\
\hline & & 0 & 1 & 2 & 3 & 4 & 5 & 6 & 7 \\
\hline \multirow{8}{*}{ 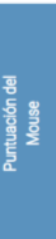 } & 0 & 1 & 1 & 1 & 2 & 3 & 4 & 5 & 6 \\
\hline & 1 & 1 & 1 & 2 & 3 & 4 & 5 & 6 & 7 \\
\hline & 2 & 1 & 2 & 2 & 3 & 4 & 5 & 6 & 7 \\
\hline & 3 & 2 & 3 & 3 & 3 & 5 & 6 & 7 & 8 \\
\hline & 4 & 3 & 4 & 4 & 5 & 5 & 6 & 7 & 8 \\
\hline & 5 & 4 & 5 & 5 & 6 & 6 & 7 & 8 & 9 \\
\hline & 6 & 5 & 6 & 6 & 7 & 7 & 8 & 8 & 9 \\
\hline & 7 & 6 & 7 & 7 & 8 & 8 & 9 & 9 & 9 \\
\hline
\end{tabular}

Fuente. Diego-Mas (2015).

Figura 5. Diagrama de puntuación tabla D del método ROSA.

\begin{tabular}{|c|c|c|c|c|c|c|c|c|c|c|}
\hline \multirow{2}{*}{\multicolumn{2}{|c|}{ TABLAD }} & \multicolumn{9}{|c|}{ Puntuación Tabla C } \\
\hline & & 1 & 2 & 3 & 4 & 5 & 6 & 7 & 8 & 9 \\
\hline \multirow{9}{*}{ 总 } & 1 & 1 & 2 & 3 & 4 & 5 & 6 & 7 & 8 & 9 \\
\hline & 2 & 2 & 2 & 3 & 4 & 5 & 6 & 7 & 8 & 9 \\
\hline & 3 & 3 & 3 & 3 & 4 & 5 & 6 & 7 & 8 & 9 \\
\hline & 4 & 4 & 4 & 4 & 4 & 5 & 6 & 7 & 8 & 9 \\
\hline & 5 & 5 & 5 & 5 & 5 & 5 & 6 & 7 & 8 & 9 \\
\hline & 6 & 6 & 6 & 6 & 6 & 6 & 6 & 7 & 8 & 9 \\
\hline & 7 & 7 & 7 & 7 & 7 & 7 & 7 & 7 & 8 & 9 \\
\hline & 8 & 8 & 8 & 8 & 8 & 8 & 8 & 8 & 8 & 9 \\
\hline & 9 & 9 & 9 & 9 & 9 & 9 & 9 & 9 & 9 & 9 \\
\hline
\end{tabular}

Fuente. Diego-Mas (2015). 
Figura 6. Diagrama de puntuación tabla E del método ROSA.

\begin{tabular}{|c|c|c|c|c|c|c|c|c|c|c|c|}
\hline \multirow{2}{*}{\multicolumn{2}{|c|}{ TABLAE }} & \multicolumn{10}{|c|}{ Puntuación Pantalla y Periféricos } \\
\hline & & 1 & 2 & $\mathbf{3}$ & 4 & 5 & 6 & 7 & 8 & 9 & 10 \\
\hline \multirow{10}{*}{$\begin{array}{l}\text { 总 } \\
\text { 旁 } \\
\text { 产 }\end{array}$} & 1 & 1 & 2 & 3 & 4 & 5 & 6 & 7 & 8 & 9 & 10 \\
\hline & 2 & 2 & 2 & 3 & 4 & 5 & 6 & 7 & 8 & 9 & 10 \\
\hline & 3 & 3 & 3 & 3 & 4 & 5 & 6 & 7 & 8 & 9 & 10 \\
\hline & 4 & 4 & 4 & 4 & 4 & 5 & 6 & 7 & 8 & 9 & 10 \\
\hline & 5 & 5 & 5 & 5 & 5 & 5 & 6 & 7 & 8 & 9 & 10 \\
\hline & 6 & 6 & 6 & 6 & 6 & 6 & 6 & 7 & 8 & 9 & 10 \\
\hline & 7 & 7 & 7 & 7 & 7 & 7 & 7 & 7 & 8 & 9 & 10 \\
\hline & 8 & 8 & 8 & 8 & 8 & 8 & 8 & 8 & 8 & 9 & 10 \\
\hline & 9 & 9 & 9 & 9 & 9 & 9 & 9 & 9 & 9 & 9 & 10 \\
\hline & 10 & 10 & 10 & 10 & 10 & 10 & 10 & 10 & 10 & 10 & 10 \\
\hline
\end{tabular}

Fuente. Diego-Mas (2015).

Se obtuvieron los ángulos de las posturas de uso frecuente en el trabajo administrativo. Posteriormente, se compararon con los criterios de referencia de Pheasant (1998) para establecer el grado de desviación que existe entre las posturas trabajo y las que se considera como aceptables. La goniometría se obtuvo mediante la medición por medio de fotografías (Ergonautas, s.f.).

Para obtener los niveles de iluminación, primeramente, se determinó el índice local (IL), y con este, se procedió a obtener el número de puntos de medición (NPM). Una vez obtenido el NPM, se calculó la distribución de los puntos de medición a lo largo y ancho de la sala. Las mediciones se efectuaron en cada uno de los puntos de medición obtenidos, a una altura de 80 centímetros o sobre la superficie del plano de trabajo. Se obtuvo el valor de uniformidad de iluminación, utilizando la aplicación de Science Journal para el uso del luxómetro (Management, s.f.).

El IL se calculó utilizando las medidas que se muestran en la tabla 1, con la siguiente fórmula:

$$
\mathrm{IL}=\frac{\mathrm{a} \times \mathrm{b}}{\mathrm{h}(\mathrm{a}+\mathrm{b})}
$$

El IL se sustituye en la fórmula a continuación, que corresponde a la variable $\mathrm{X}$ redondeada al entero.

$$
\mathrm{NPM}=(x+2)^{2}
$$


Tabla 1. Descripción correspondiente del espacio a evaluar.

\begin{tabular}{|c|l|}
\hline Ítem & Descripción \\
\hline $\mathrm{a}$ & Largo de la sala \\
\hline $\mathrm{b}$ & Ancho de la sala \\
\hline $\mathrm{c}$ & Alto total de la sala \\
\hline $\mathrm{d}$ & Distancia desde el techo a la luminaria (cero si está embutida) \\
\hline $\mathrm{e}$ & Altura de la superficie de trabajo \\
\hline $\mathrm{h}$ & $\begin{array}{l}\text { Distancia vertical entre la superficie de trabajo y la luminaria (se determina restando } \\
\text { a la altura total la suma de d+e) }\end{array}$ \\
\hline
\end{tabular}

El resultado de los NPM (varía desde 9 hasta 36 puntos), los cuales son distribuidos considerando el ancho y largo del espacio evaluar. Para calcular los puntos de medición a lo ancho y largo del espacio a evaluar, respectivamente, donde $\mathrm{N}$ total corresponde a NPM, se aplicaron las siguientes fórmulas:

$$
N \text { ancho }=\sqrt{\frac{N \text { total }}{\text { Largo (a) }} \times \text { Ancho (b) }}
$$

$$
\mathrm{N} \text { largo }=\mathrm{N} \text { ancho } \times \frac{\operatorname{Largo}(\mathrm{a})}{\text { Ancho }(\mathrm{b})}
$$

Para determinar si la distribución de la luz es adecuada en el espacio evaluado se aplicó el criterio de Uniformidad de Iluminación (UI), donde UI = iluminancia mínima medida/ iluminancia media medida. El valor resultante debe ser mayor o igual o,80; si es menor indica que la iluminación no está distribuida en forma uniforme.

\section{Resultados y discusión}

Carga organizacional de trabajo y esfuerzo cardiovascular

El análisis del estudio de tiempos se realizó en tres días laborales diferentes, con el propósito de identificar si las variabilidades de las actividades sobrepasan la capacidad de carga de trabajo en el trabajador. Las figuras 7 y 8 muestran algunas de las actividades a evaluar. 
Figura 7. Actividades en oficina.

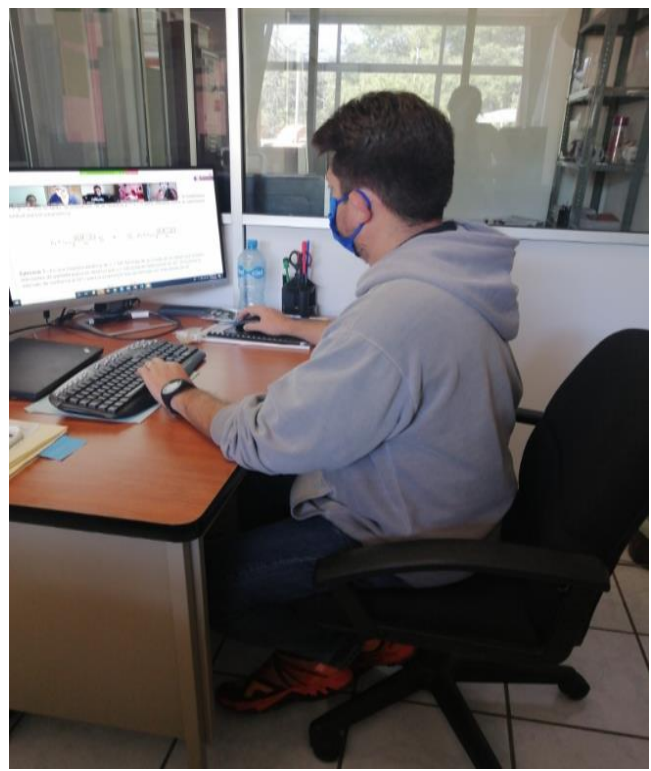

Figura 8. Actividades en campo.

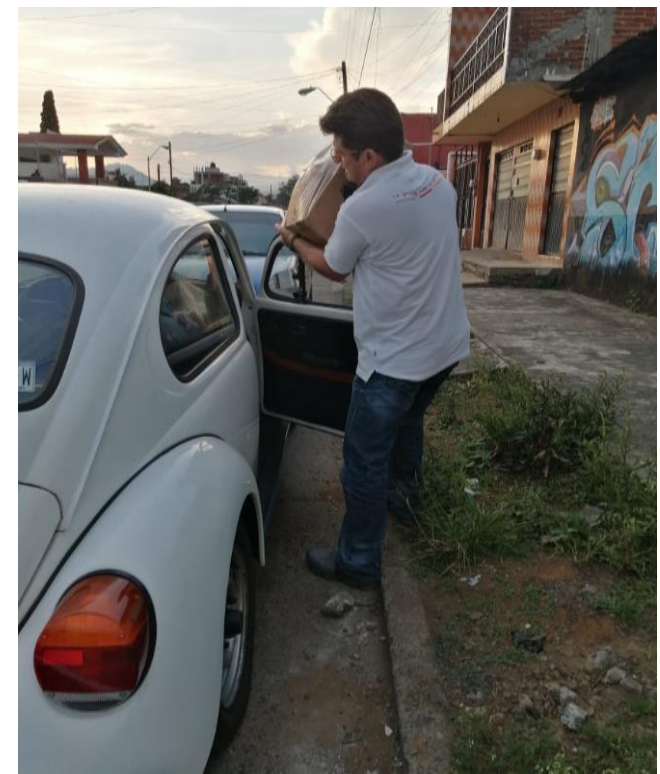

Las actividades en el desarrollo de los análisis están concentradas en las tablas 2 y 3.

Tabla 2. Actividades principales y secundarias del análisis.

\begin{tabular}{|l|l|}
\hline Actividades & Descripción \\
\hline Administrativas & $\begin{array}{l}\text { Atención a clientes de manera personal y vía telefónica, llamadas } \\
\text { telefónicas, realización y respuesta de correos electrónicos, captura de los } \\
\text { servicios solicitados por los clientes y entrega de los informes de } \\
\text { resultados. }\end{array}$ \\
\hline Asesoría residentes & Atención de alumnos que están realizando su estadía profesional. \\
\hline Clase en línea & Impartición de clase mediante plataformas electrónicas. \\
\hline Conducir & Desplazamiento por medio de vehículo. \\
\hline Entrega de paquete & Solicitud de servicios a empresas de paquetería y mensajería. \\
\hline $\begin{array}{l}\text { Entrega de material } \\
\text { de muestreo }\end{array}$ & Entrega de insumos de muestreo a los clientes. \\
\hline $\begin{array}{l}\text { Firma de } \\
\text { documentos }\end{array}$ & Firma de oficio de índole académico. \\
\hline $\begin{array}{l}\text { Recolección } \\
\text { muestras }\end{array}$ & Recoger muestras en los domicilios de los clientes. \\
\hline Trámite bancario & Realización de pagos y depósitos bancarios. \\
\hline
\end{tabular}

Tabla 3. Actividades cotidianas no laborales.

\begin{tabular}{|l|l|}
\hline Actividades & Descripción \\
\hline Aseo personal & Higiene personal. \\
\hline Comida & Ingesta de alimentos. \\
\hline Sin actividad & La no realización de actividades laborales. \\
\hline
\end{tabular}

Los valores del primer análisis muestran el tiempo que invirtió el trabajador desde la hora que se despertó hasta la hora en que terminó de realizar su última actividad laboral 
(tabla 4). En actividades principales invierte el 77,34\% de los 728 minutos evaluados. En actividades secundarias y en pausas invierte $3,57 \%$ y $19,09 \%$ respectivamente (tabla 5 ).

Durante el análisis de tiempos, de manera simultánea se midieron los latidos cardíacos del trabajador ya que estos valores ayudan a determinar; si para efectos de la realización de las actividades, existe fatiga física por medio de la obtención del \% CC. En el primer día analizado la frecuencia cardíaca promedio fue de 86 palpitaciones por minuto (ppm). Tomando los datos personales del trabajador que se muestran en figura 9, se determinó que el \% CC es de 25,61 y el desgaste generado representó un gasto energético de 2.167 kcal.

Tabla 4. Primer día de análisis.

\begin{tabular}{|c|c|c|c|c|}
\hline Hora inicio & Hora término & Actividad & Minutos & \% \\
\hline $7: 33$ & $7: 52$ & Conducir & 19 & 2,61 \\
\hline $7: 52$ & $9: 49$ & Clase en línea & 117 & 16,07 \\
\hline $9: 49$ & $10: 38$ & Actividades administrativas & 49 & 6,73 \\
\hline $11: 34$ & $11: 47$ & Conducir & 13 & 1,79 \\
\hline $13: 25$ & $13: 37$ & Conducir & 12 & 1,65 \\
\hline $13: 37$ & $14: 00$ & Actividades administrativas & 23 & 3,16 \\
\hline $14: 00$ & $15: 40$ & Clase en línea & 100 & 13,74 \\
\hline $15: 40$ & $17: 35$ & Actividades administrativas & 115 & 15,80 \\
\hline $17: 35$ & $17: 51$ & Conducir & 16 & 2,20 \\
\hline $17: 51$ & $18: 29$ & Recolección de muestras & 38 & 5,22 \\
\hline $18: 29$ & $18: 50$ & Conducir & 21 & 2,88 \\
\hline $18: 50$ & $18: 56$ & Actividades administrativas & 6 & 0,82 \\
\hline $18: 56$ & $19: 02$ & Conducir & 6 & 0,82 \\
\hline $19: 02$ & $19: 08$ & Entrega de paquete & 6 & 0,82 \\
\hline $19: 08$ & $19: 12$ & Conducir & 4 & 0,55 \\
\hline $19: 12$ & $19: 30$ & Actividades administrativas & 18 & 2,47 \\
\hline
\end{tabular}

Tabla 5. Resumen de actividades primer día de análisis.

\begin{tabular}{|c|c|c|}
\hline Actividad & Tiempo en minutos & \% \\
\hline Principal & 563 & 77,34 \\
\hline Secundaria & 26 & 3,57 \\
\hline Pausas & 139 & 19,09 \\
\hline Total & 728 & 100 \\
\hline
\end{tabular}


Figura 9. Datos trabajador evaluado.

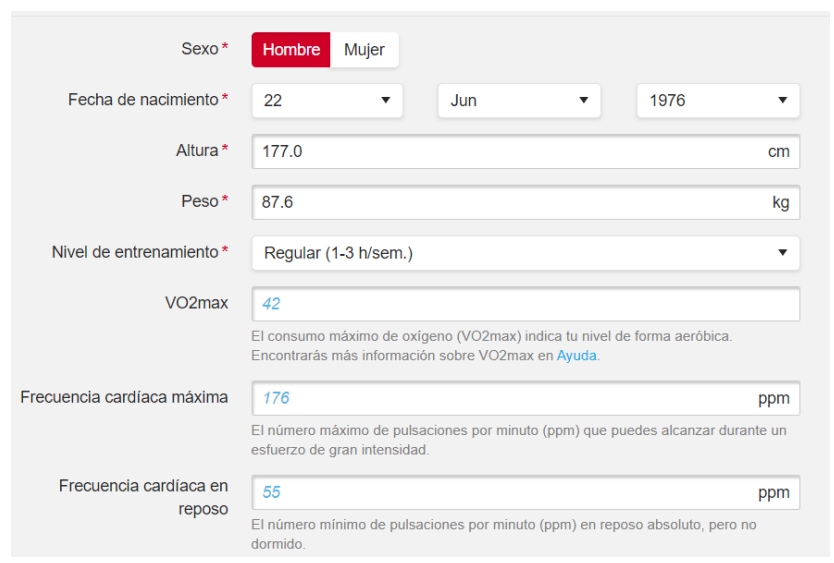

Nota. Extraído Polar Flow.

En la figura 10 se muestra la variabilidad de las palpitaciones por minuto que tuvo el trabajador durante la realización de sus actividades en el primer día analizado.

Figura 10. Palpitaciones en primer día analizado.

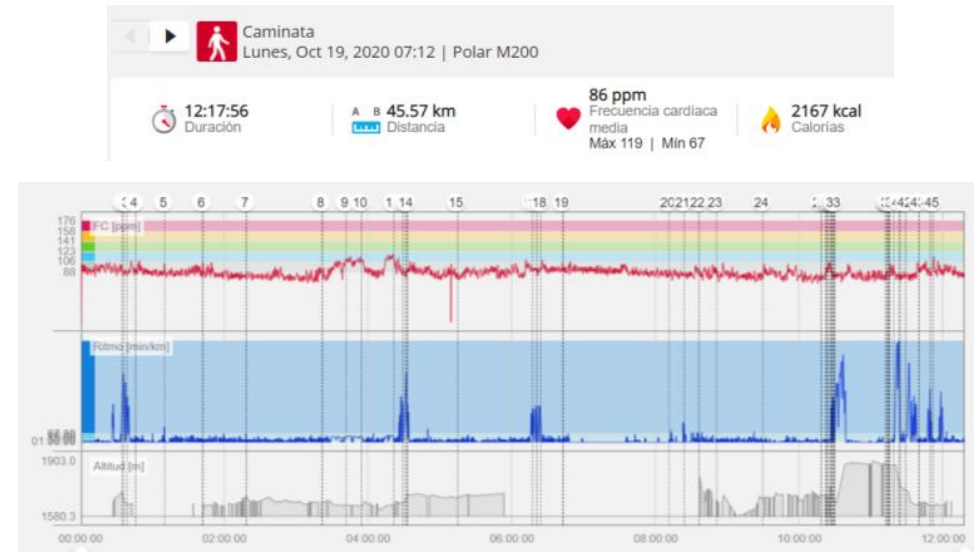

Nota. Extraído Polar Flow.

Los valores del segundo análisis que se observan en la tabla 6, muestran que en actividades principales invierte el 56,26\% de los 855 minutos evaluados. En actividades secundarias y en pausas invierte $17,43 \%$ y $26,32 \%$ respectivamente. Llama la atención que pese a que fueron más minutos evaluados el porcentaje de tiempo invertido en la realización de actividades principales disminuyó, como se observa en la tabla 7.

En el segundo día analizado la frecuencia cardíaca promedio fue de $94 \mathrm{ppm}$. Con esta frecuencia se determinó un \% CC de 32,23 y el desgaste generado representó un gasto energético fue de $3.764 \mathrm{kcal}$. La figura 11 muestra la variabilidad de las palpitaciones por minuto que tuvo el trabajador durante la realización de sus actividades, en el segundo día analizado. Es importante destacar que la ppm máxima de este día analizado fue de 126. 
Tabla 6. Segundo día de análisis.

\begin{tabular}{|c|c|c|c|c|}
\hline Hora inicio & Hora término & Actividad & Minutos & \% \\
\hline $6: 05$ & $6: 18$ & Conducir & 13 & 1,79 \\
\hline $6: 18$ & $6: 19$ & Recolección de muestras & 1 & 0,14 \\
\hline $6: 19$ & $6: 31$ & Conducir & 12 & 1,65 \\
\hline $6: 31$ & $7: 12$ & Actividades administrativas & 41 & 5,63 \\
\hline $7: 12$ & $7: 18$ & Conducir & 6 & 0,82 \\
\hline $7: 18$ & $7: 27$ & Entrega de paquete & 9 & 1,24 \\
\hline $7: 27$ & $7: 32$ & Conducir & 5 & 0,69 \\
\hline $7: 32$ & $9: 28$ & Clase en línea & 116 & 15,93 \\
\hline $9: 28$ & $11: 00$ & Actividades administrativas & 32 & 4,40 \\
\hline $11: 00$ & $11: 17$ & Conducir & 17 & 2,34 \\
\hline $11: 17$ & $11: 49$ & Trámite bancario & 32 & 4,40 \\
\hline $11: 49$ & $11: 58$ & Conducir & 9 & 1,24 \\
\hline $14: 27$ & $14: 40$ & Conducir & 13 & 1,79 \\
\hline $14: 40$ & $15: 00$ & Actividades administrativas & 20 & 2,75 \\
\hline $15: 00$ & $15: 37$ & Asesoría residente & 37 & 5,08 \\
\hline $15: 37$ & $17: 41$ & Actividades administrativas & 4 & 0,55 \\
\hline $17: 41$ & $18: 00$ & Conducir & 19 & 2,61 \\
\hline $18: 00$ & $18: 02$ & Recolección de muestras & 2 & 0,27 \\
\hline $18: 02$ & $18: 22$ & Conducir & 20 & 2,75 \\
\hline $18: 22$ & $19: 02$ & Actividades administrativas & 30 & 4,12 \\
\hline $19: 02$ & $19: 09$ & Conducir & 7 & 0,96 \\
\hline $19: 09$ & $19: 19$ & Entrega de paquete & 10 & 1,37 \\
\hline $19: 19$ & $19: 24$ & Conducir & 5 & 0,69 \\
\hline $19: 24$ & $19: 45$ & Actividades administrativas & 21 & 2,88 \\
\hline & & & & \\
\hline
\end{tabular}

Tabla 7. Resumen de actividades segundo día de análisis.

\begin{tabular}{|c|c|c|}
\hline Actividad & Tiempo en minutos & \% \\
\hline Principal & 481 & 56,26 \\
\hline Secundaria & 149 & 17,43 \\
\hline Pausas & 225 & 26,31 \\
\hline Total & 855 & 100 \\
\hline
\end{tabular}


Figura 11. Palpitaciones en segundo día analizado.

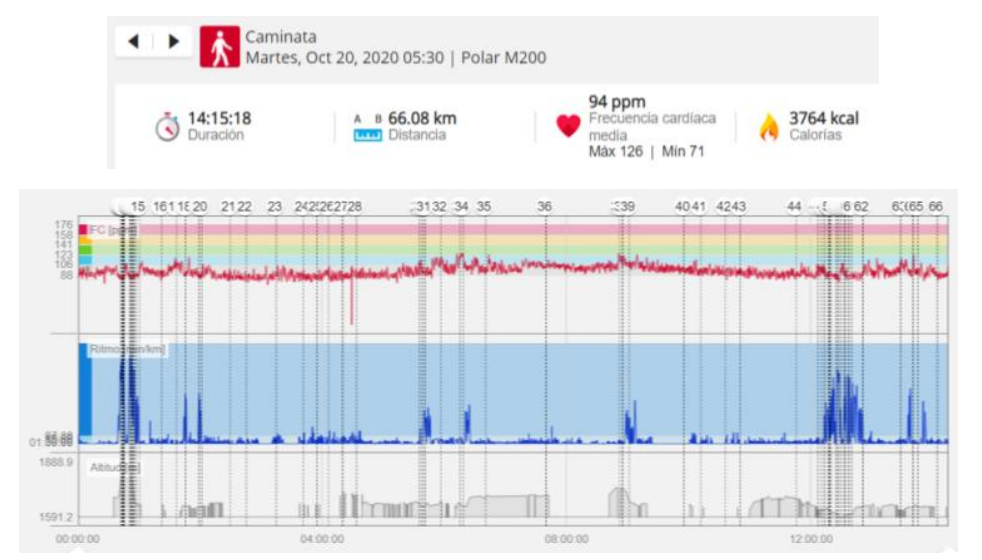

Nota. Extraído Polar Flow.

Para el tercer análisis que se observa en la tabla 8, se obtuvo que en las actividades principales invierte el $77,41 \%$ de los 735 minutos evaluados. En actividades secundarias y en pausas invierte 19,05\% y 3,54\% respectivamente. En este análisis se destaca que el porcentaje de tiempo invertido en actividades secundarias incrementó y disminuyó el porcentaje de tiempo invertido en pausas, estos valores, totalmente opuestos a los obtenidos en el primer y segundo análisis, como se muestra en la tabla 9.

Para el tercer día analizado la frecuencia cardíaca promedio fue de $91 \mathrm{ppm}$. Con esta frecuencia se determinó un \% CC de 29,75 y el desgaste generado representó un gasto energético de $2.887 \mathrm{kcal}$.

Tabla 8. Tercer día de análisis.

\begin{tabular}{|c|c|c|c|c|}
\hline Hora inicio & Hora término & Actividad & Minutos & \% \\
\hline $7: 37$ & $7: 47$ & Conducir & 10 & 1,37 \\
\hline $7: 47$ & $7: 56$ & Actividades administrativas & 9 & 1,24 \\
\hline $7: 56$ & $8: 00$ & Conducir & 4 & 0,55 \\
\hline $8: 00$ & $8: 11$ & Entrega de paquete & 11 & 1,51 \\
\hline $8: 11$ & $8: 27$ & Conducir & 16 & 2,20 \\
\hline $8: 27$ & $9: 00$ & Asesoría residente & 33 & 4,53 \\
\hline $9: 00$ & $9: 30$ & Clase en línea & 30 & 4,12 \\
\hline $9: 30$ & $11: 19$ & Actividades administrativas & 109 & 14,97 \\
\hline $11: 19$ & $11: 47$ & Firma documentos (recorrido) & 28 & 3,85 \\
\hline $11: 47$ & $11: 55$ & Actividades administrativas & 8 & 1,10 \\
\hline $11: 55$ & $12: 13$ & Conducir & 18 & 2,47 \\
\hline $12: 13$ & $13: 22$ & Actividades administrativas & 69 & 9,48 \\
\hline $13: 48$ & $13: 58$ & Conducir & 10 & 1,37 \\
\hline $13: 58$ & $15: 40$ & Clase en línea & 102 & 14,01 \\
\hline $15: 40$ & $17: 45$ & Actividades administrativas & 5 & 0,69 \\
\hline $17: 45$ & $18: 07$ & Conducir & 22 & 3,02 \\
\hline $18: 07$ & $18: 09$ & Entrega material de muestreo & 2 & 0,69 \\
\hline
\end{tabular}




\begin{tabular}{|c|c|c|c|c|}
\hline $18: 09$ & $18: 12$ & Conducir & 3 & 0,41 \\
\hline $18: 12$ & $18: 14$ & Recolección de muestras & 2 & 0,27 \\
\hline $18: 14$ & $18: 40$ & Conducir & 26 & 3,57 \\
\hline $18: 40$ & $19: 49$ & Actividades administrativas & 9 & 1,24 \\
\hline $18: 49$ & $18: 55$ & Conducir & 6 & 0,82 \\
\hline $18: 55$ & $19: 00$ & Entrega de paquete & 5 & 0,69 \\
\hline $19: 00$ & $19: 05$ & Conducir & 5 & 0,69 \\
\hline $19: 05$ & $19: 32$ & Actividades administrativas & 27 & 3,71 \\
\hline
\end{tabular}

Tabla 9. Resumen de actividades tercer día de análisis.

\begin{tabular}{|c|c|c|}
\hline Actividad & Tiempo en minutos & \% \\
\hline Principal & 569 & 77,41 \\
\hline Secundaria & 140 & 19,05 \\
\hline Pausas & 26 & 3,54 \\
\hline Total & 735 & 100 \\
\hline
\end{tabular}

La figura 12 muestra la variabilidad de las palpitaciones por minuto que tuvo el trabajador durante la realización de sus actividades, en el segundo día analizado. La ppm máxima en el análisis de este día fue la mayor de los tres estudios con 138.

Figura 12. Palpitaciones en tercer día analizado.

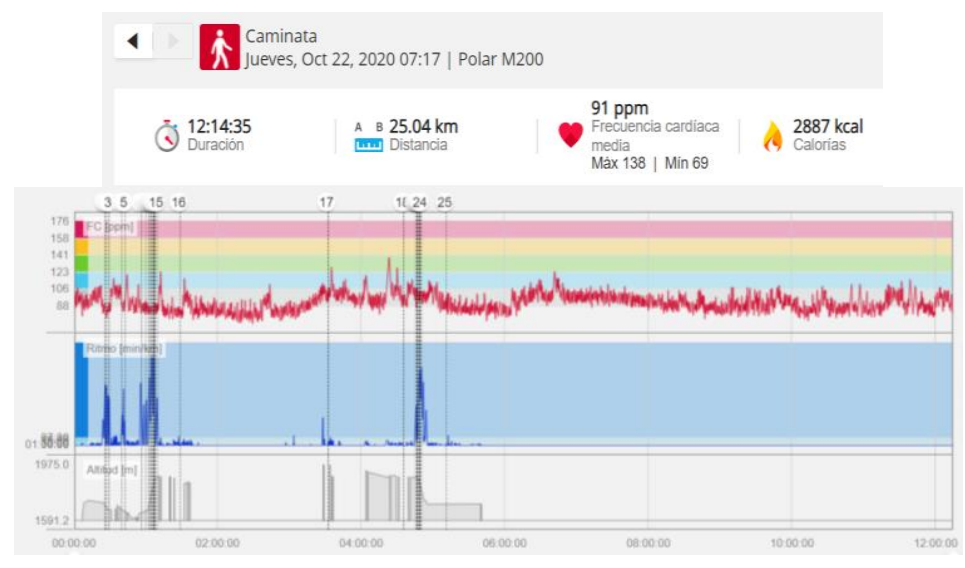

Nota. Extraído Polar Flow.

Conforme avanzan los días de actividad, el cuerpo resiente el desgaste acumulado y si no se toman los reposos necesarios para la recuperación se podrán presentar desórdenes músculo-esqueléticos por fatiga laboral (Apud \& Meyer, 2009).

Características del mobiliario y posturas adoptadas durante el trabajo de tipo administrativo

Para aplicar el método ROSA se observó el puesto de trabajo mientras el trabajador desarrolla sus actividades docentes (figura 13). Asignados los valores a cada uno de los elementos del puesto: silla, pantalla, teclado, mouse y teléfono, se calificaron los diferentes elementos del puesto con ayuda de los diagramas de puntuación. 
Figura 13. Realización de actividades docentes.
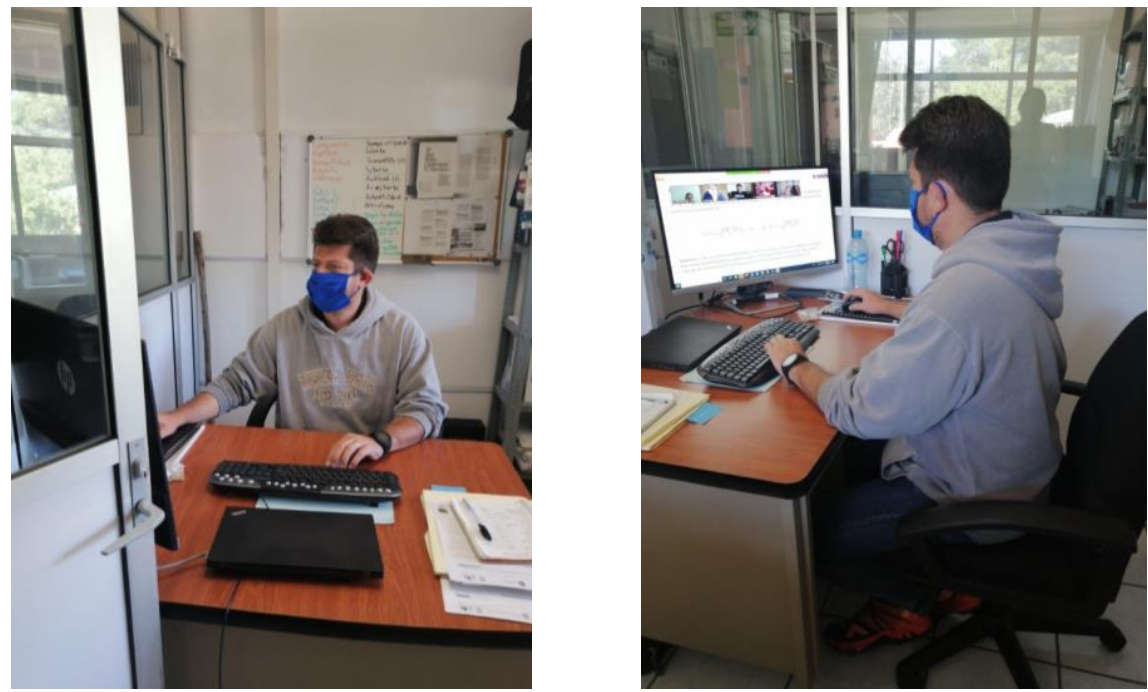

El resultado de la evaluación del puesto de trabajo con el método ROSA fue de 8 como se observa en la tabla 10. Con respecto al nivel de actuación que proporciona el método ROSA, es necesario efectuar mejoras en las variables analizadas (silla, pantalla, periféricos), buscando el punto óptimo de la postura de trabajo.

Tabla 10. Resultado de la evaluación del método ROSA.

\begin{tabular}{|l|c|}
\hline \multicolumn{1}{|c|}{ Tablas } & Puntuación \\
\hline A Reposabrazos + respaldo + altura asiento + profundidad de asiento & 7 \\
\hline B Teléfono + pantalla & 2 \\
\hline C Mouse + teclado & 4 \\
\hline D Teléfono + pantalla + teclado + mouse & 4 \\
\hline E Silla + teléfono + pantalla + teclado + mouse & 8 \\
\hline
\end{tabular}

Se determinó el grado de desviación que existe entre las condiciones de trabajo y lo que se considera aceptable. La evaluación está orientada a definir posturas de menor riesgo para el sistema músculo-esquelético, que sean funcionales y cómodas.

En la figura 14a se presentan algunos ángulos de comodidad referidos en la literatura (Pheasant, 1998). Como se pueda observar, las posturas cómodas no son ángulos específicos, sino rangos de desplazamiento que las personas puedan adoptar para alcanzar una condición de confort.

Considerando los ángulos de comodidad y los registrados en el trabajador, se toman como ejemplo dos segmentos corporales. El primero es la postura del tronco. Las referencias de ángulos de comodidad indican que la tarea en postura sentado, debería estar en extensión entre $0^{\circ}$ y $15^{\circ}$. Como se puede apreciar en la figura $14 \mathrm{~b}$, el trabajador está a $16^{\circ}$, pero hacia la parte anterior de la vertical, está en flexión y fuera de los ángulos de comodidad. 
Figura 14. a) Ángulos de comodidad (Pheasant, 1998); b) Ángulos registrados con goniometría del trabajador en su área de trabajo.
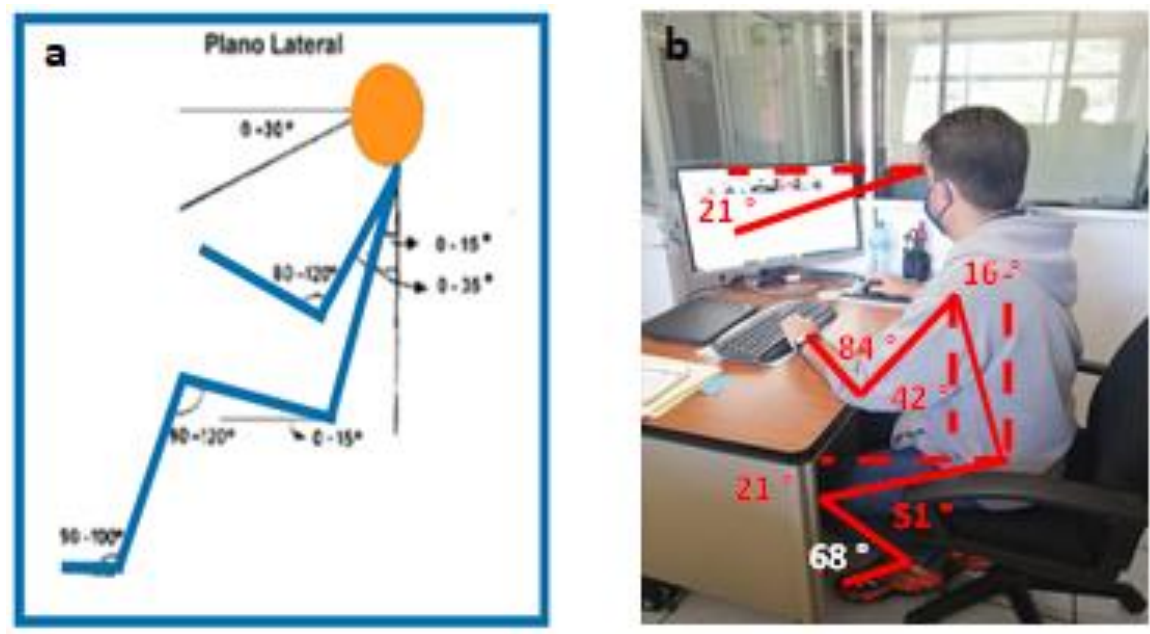

En el segmento que corresponde a la articulación de la cadera las referencias de los ángulos de comodidad indican que el muslo debería estar entre $0^{\circ}$ y $15^{\circ}$ respecto de la horizontal. Al comparar las referencias con los datos obtenidos con la técnica de goniometría, se observa que el muslo está a $21^{\circ}$ bajo la horizontal, por lo tanto, fuera del ángulo comodidad. Sólo haciendo mención de los dos ejemplos, se entiende la existencia de sobrecarga postural.

\section{Niveles de iluminación}

Los valores (ítems de la tabla 1) del espacio evaluado fueron: $a=2,73 \mathrm{~m} ; \mathrm{b}=2,47 \mathrm{~m} ; \mathrm{c}=2,48$ $\mathrm{m} ; \mathrm{d}=\mathrm{O} \mathrm{m} ; \mathrm{e}=0,74 \mathrm{~m} ; \mathrm{h}=1,74 \mathrm{~m}$. El resultado del NPM fue de 9 puntos. Los rectángulos de los puntos de medición quedaron con las dimensiones de o,82 $\mathrm{m}$ de ancho por 0,91 $\mathrm{m}$ de largo. Se tomó la medición de iluminación en cada uno de los nueve puntos. Los valores obtenidos fueron: 66, 88, 139, 82, 94, 147, 50, 68 y 97 luxes, como se muestra en la figura 15.

Con respecto a las mediciones obtenidas, el valor más alto fue 147 luxes, el valor más bajo fue 50 luxes y el valor promedio 92 luxes. El Decreto Supremo 594 (1999) de la legislación chilena y la NOM-025-STPS-2008 norma mexicana, estipulan que para el trabajo prolongado con requerimiento moderado sobre la visión es recomendable un mínimo de iluminancia de 300 luxes. El valor de Uniformidad de Iluminación resultó de 0,54.

Figura 15. Valores de la medición de la iluminación expresada en luxes.

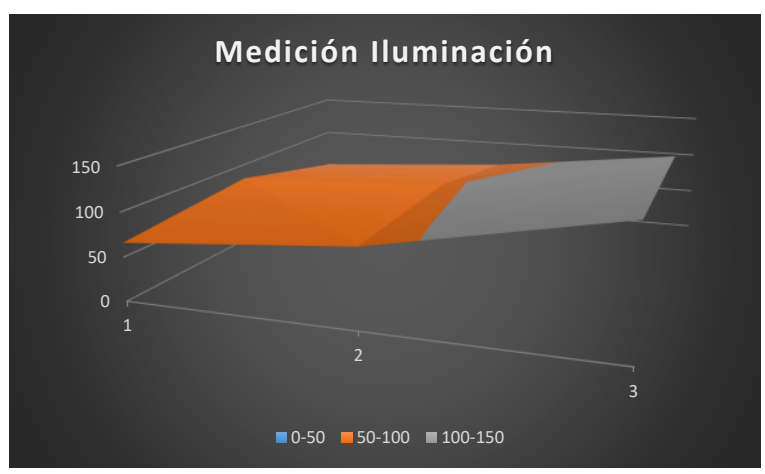




\section{Conclusiones}

Un valor de $80 \%$ o mayor en la suma de las actividades principales y secundarias es considerado como sobredemanda laboral. Los resultados de los estudios de tiempos del día 1 y el día 3 muestran porcentajes mayores al $80 \%$ en la realización de ambas actividades, preocupando el 96,96\% obtenido en el estudio del día 3. También es conveniente tener en cuenta otras consideraciones para tomar la decisión si la actividad es o no demandante, como lo son los \%CC peak (máximos). Los valores de las ppm máximos 119, 126 y 138 da como resultado \%CC mayores a $40 \%$. Se pudiera decir que para el caso de estudio, no es trabajo pesado, pero los estudios realizados superan las 8 horas de actividad laboral y sobresale que, en el tercer día de estudio se obtuvo la frecuencia cardíaca mayor con 138 ppm.

Considerar los ángulos de comodidad y calcular la desviación de las características del puesto evaluado dieron como resultado la existencia de sobrecarga postural y el nivel de actuación que entrega el método ROSA sugiere intervenir de manera inmediata.

El criterio de uniformidad de iluminación, como su nombre lo indica, mostró que en el área de trabajo no existe uniformidad en las luminarias utilizadas y además no cuenta con el mínimo requerido de iluminancia. Esto es muy común, debido a que no se realiza planeación para los espacios administrativos, y estos se configuran conforme se van requiriendo. Por consiguiente, es recomendable prever qué espacios se considerarán en algún momento, como cubículos de trabajo, y así otorgar la iluminación idónea para el lugar de trabajo. De las mediciones obtenidas el valor más alto fue de 147 luxes y el promedio fue de 92 luxes, muy por debajo de lo recomendado.

Sin duda, el estudio de tiempos, la evaluación de los ángulos de las posturas laborales, y la medición de la iluminación, evidencian condiciones disergonómicas en muchas de las actividades cotidianas que realiza el trabajador. Es momento de seguir insistiendo que las intervenciones ergonómicas, sin duda, son el escape para disminuir la epidemiología de las enfermedades laborales.

\section{Recomendaciones}

Es importante considerar que las actividades laborales no deben ser mayores a 8 horas diarias, debido a que trabajar largas horas causa cansancio, estrés, frustración y baja productividad.

Se sugiere invitar al trabajador a mejorar sus hábitos de postura, aunado a mejorar los ángulos del equipo de cómputo y los periféricos, además de proponer el uso de una silla que otorgue comodidad postural.

Es importante establecer directrices para el mobiliario de trabajo en donde los trabajadores permanecen sentados; lo ideal es que sea ajustable. Normalmente, los diseños se generan tomando en cuenta las personas de mayor tamaño (95 percentil) en el entendido que al existir espacio suficiente se pueden hacer modificaciones para acomodar a las personas más pequeñas. El 95 percentil es válido para las superficies de trabajo, no obstante, para el diseño de los asientos (sillas) dependerá del ajuste para que los trabajadores se adapten a su puesto de trabajo. 
Se recomienda que las sillas de trabajo tengan una profundidad máxima de $40 \mathrm{~cm}$, el ancho debe tener por lo menos $46 \mathrm{~cm}$, la regulación de la altura debe tener un rango entre 38 cm y $47 \mathrm{~cm}$, la atura de los apoya brazos conviene tener un ajuste entre $59 \mathrm{~cm} \mathrm{y} 73 \mathrm{~cm}$, aunque es conveniente estudiar cada caso en particular, y que la altura del respaldo tenga como mínimo $75 \mathrm{~cm}$ y máximo 112,5 $\mathrm{cm}$ medidos desde el suelo (Instituto de Salud Pública de Chile, 2016).

Los escritorios se recomiendan sean ajustables en su altura entre $60 \mathrm{~cm} \mathrm{y} 73 \mathrm{~cm}$. La profundidad debe ser de $80 \mathrm{~cm}$; con esta medida se asegura la distancia ideal entre la pantalla y el trabajador. El ancho recomendado es de $160 \mathrm{~cm}$ y una forma de "L"; esto permitirá tener el espacio adecuado tanto para el computador como para escribir.

Con respecto a la iluminación, es recomendable analizar la factibilidad de implementar luz artificial para mejorar los niveles de iluminación. Sin embargo, reubicar los muebles y los equipos de trabajo ayudará a que la luz natural se aproveche de mejor manera. Posteriormente, es conveniente realizar una reevaluación para verificar si con las modificaciones se obtuvieron niveles de iluminación y uniformidad adecuados 


\section{Referencias}

Álvarez, F. J. (2009). Ergonomía y psicosociología aplicada. Manual para la formación del Especialista. Lex Nova.

Apud, E., \& Meyer, F. (2009). Ergonomía para la industria minera. Universidad de Concepción de Chile.

Decreto Supremo 594. (1999). Aprueba el reglamento sobre condiciones sanitarias y ambientales básicas en los lugares. http://www.ist.cl/wpcontent/uploads/2016/08/DECRETO-SUPREMO-594-ACTUALIZADO-2019.pdf

Departamento de Ergonomía de la Universidad de Concepción y Sociedad Chilena de Ergonomía. (2020). Entorno ergonómico en situación de pandemia. https://www.sochergo.cl/wpcontent/uploads/2020/08/ERGONOMIA-Y-TELETRABAJO-UDEC-SOCHERGO-2020.pdf

Diego-Mas, J. A. (2015). Evaluación de puestos de trabajo de oficinas mediante el método ROSA. Universidad Politécnica de Valencia. http://www.ergonautas.upv.es/metodos/rosa/rosaayuda.php

Ergonautas. (s.f.). RULER - Medición de ángulos en fotografias. Universidad Politécnica de Valencia. Consultado el 31 de octubre de 2020. http://www.ergonautas.upv.es/herramientas/ruler/ruler.php

Gobierno de México. (2020, 28 de Octubre). Todo sobre el COVID-19. https://coronavirus.gob.mx/informacion-accesible/

Instituto de Salud Pública de Chile. (2016). Guía de ergonomía . Identificación y control de factores de riesgo en el trabajo de oficina y el uso de computador. Obtenido de https://www.ispch.cl/salud-de-los-trabajadores/publicaciones-de-referencia/ergonomia/

Maestre, D. G. (2015). Ergonomía y Psicosociología. FC Editorial.

Management, I. J. (s.f.). Sensors on your device. Consultado el 5 de octubre de 2020. https://support.google.com/sciencejournal/?hl=en

NOM-025-STPS-2008. (s.f.). Condiciones de iluminación en los centros de trabajo. http://www.dof.gob.mx/normasOficiales/3581/stps/stps.htm

Organización Internacional del Trabajo. (1998). Enciclopedia de salud y seguridad en el trabajo. https://www.insst.es/tomo-i

Pheasant, S. B. (1998). Body space: Anthropometry, ergonomics and the design of work. Taylor and Francis. 